\title{
Einige Überlegungen zum «ungebremsten» Kostenanstieg im Gesundheitswesen*
}

\author{
A. Oberle
}

\section{Zur Ausgangslage}

Seit gut 30 Jahren etwa beobachten wir einen im Vergleich zu den allgemeinen Lebenskosten unverhältnismässigen Anstieg der Kosten für unser Gesundheitswesen. Nicht wenige Politiker und Journalisten sprechen gar von einer Kostenexplosion und finden dafür Erklärungen

- für den Spitalbereich im Fehlen interkantonaler Zusammenarbeit und ökonomischer Aufgabenteilung der Spitäler und Krankenanstalten oder in ihrer mangelhaften betriebswirtschaftlichen Führung mit unangebrachtem Perfektionismus oder gar Luxus und oft nicht ausgelasteten Einrichtungen;

- für den Kostenanstieg in der ambulanten medizinischen Betreuung machen insbesondere die Krankenversicherungen die Anspruchshaltung ihrer Versicherten, aber unverhohlen auch das Gewinnstreben der immer zahlreicheren Ärzte verantwortlich.

Seit etlichen Jahren wird ja nun versucht, Gegensteuer zu geben. So mit der Einführung einer praktisch alle Bedürfnisse abdeckenden obligatorischen Grundversicherung, mit Auslichtung der Spitallandschaft, Änderung in der Medikamentendistribution, Revisionen im Krankenversicherungsgesetz, wobei jetzt gar die Aufhebung des Kontrahierungszwanges (= Vertragsfreiheit) für die Krankenversicherer geplant ist.

Zudem stehen Initiativen ins Haus, die die Grundversicherung auf das im eigentlichen Sinne Notwendige beschränken und Struktur, Funktion und Ökonomie der beteiligten Versicherungen vereinfachen und kostengünstiger machen möchten.

Vor jeder Therapie, soll sie angemessen sein, muss eine Diagnose stehen.

Der Schlachtruf «Wir müssen die Kosten in den Griff bekommen!» ist zwar verständlich, aber allein wenig hilfreich, wenn man nicht weiss, woher diese Kosten kommen und ob überhaupt und wie sie gebändigt werden können.

Eine Auslegeordnung ist jedenfalls unvermeidlich.

In einer bemerkenswerten Darlegung [1] hat Kollege R. Streit, Burgdorf, Präsident der Ärztegesellschaft des Kantons Bern, etwa 20 Faktoren aufgeführt, die zu einem überdurchschnittlichen Kostenwachstum im Gesundheitswesen beitragen.

In allgemeinerer Form hatte ich diese Faktoren schon 1978 angedeutet [2]. Seither haben sich auch für mich die kostentreibenden Elemente noch viel deutlicher abgezeichnet, so dass die These aufgestellt werden kann:

Es ist eine Illusion zu glauben, der Kostenaufwand im Gesundheitswesen lasse sich in unsern Breitengraden und in absehbarer Zeit zum allgemeinen Lebenskostenanstieg parallel halten oder gar senken.

Allein schon aus dem einfachen Grund, weil ärztliche und pflegerische Tätigkeit weder normiert, mechanisiert oder gar automatisiert werden kann, wie viele Komponenten im kostenbestimmenden Warenkorb unserer Lebensgrundlagen.

Sie bleibt immer personalintensive Massarbeit mit Kopf und Hand, was in allen Bereichen bekanntlich kostspieliger bleibt als industriell gefertigte Produkte oder gesteuerte Anlagen.

Hierzu kommen nun aber wesentliche zusätzliche Faktoren, die den Kostenbogen weiter erhöhen. Es würde zu weit führen, sie alle im Detail darzustellen. Einige können nur angedeutet werden:

Die Zunahme der mittleren Lebenserwartung der Menschen in der westlichen Welt.

Dank besserer Lebensbedingungen, aber auch dank der Erfolge der Medizin hat sich die mittlere Lebenserwartung in den letzten hundert Jahren um rund 30 Jahre erhöht und liegt jetzt, etwas geschlechtsabhängig, zwischen 80 und 83 Jahren.

Schon durch rein natürliche Alterung und Abnutzung sind aber 70- bis 90jährige oft von Mehrfachkrankheiten betroffen, womit diese Altersstufe, insbesondere in ihrer letzten Lebensphase, in medizinischer Hinsicht sehr kostenträchtig wird. Hierzu kommt die in den letzten Jahren stark steigende Zahl von Altersdemenzen verschiedener Art mit hohem pflegerischem und medizinischem Aufwand. Trotzdem sind aber auch in diesem Alter noch grössere Interventionen, wie zum Beispiel Staroperationen bei 80jährigen oder Hüftgelenksoperationen bei 90jährigen mit Schenkelhalsfraktur, die bei weitem kostengünstigere Lösung als ihre Belassung 
in Invalidität, Immobilität und Pflegeabhängigkeit, ganz abgesehen von der Gewährung menschlicher Lebensqualität für die Betroffenen.

Weiterhin unabsehbar in seinen finanziellen Auswirkungen bleibt der mit der Entwicklung der Naturwissenschaften und Informatik immer schneller und weiter laufende Fortschritt des medizinischen Wissens, und die darauf beruhenden therapeutischen Möglichkeiten.

Es kann ruhig die Behauptung gewagt werden, dass die medizinische Wissenschaft in den letzten 50-60 Jahren zu mehr Erkenntnissen und Möglichkeiten gekommen ist, als während der ganzen vorausgehenden Menschheitsgeschichte.

Mikrobiologische, biochemische und pharmazeutische Einsichten, die Aufklärung der genetischen Struktur des Menschen, bildgebende und endoskopische Diagnoseverfahren bis hin zum «gläsernen» Menschen ergeben heute Möglichkeiten, die noch vor wenigen Jahren undenkbar waren. Bis anhin unheilbare Krankheiten können geheilt oder erträglich gehalten, schwerste Unfallfolgen beherrscht oder in Heilung, allenfalls mit Defekt, übergeführt werden, was dann verständlicherweise weiteren pflegerischen oder sozialen Aufwand nach sich zieht.

Fortschritte in der Medizin können teilweise auch den weitgehenden Wegfall der natürlichen Auslese oder Selektion kompensieren und damit das Überleben von mit erblicher Krankheit belasteten Individuen ermöglichen, können sie gar ins Reproduktionsalter bringen, was zu einer Folgekette medizinischer und sozialer Fürsorge führen kann. Auch ästhetisch-restaurative Behandlungen sind vermehrt möglich und dürfen, meist auch zu Recht, gefordert werden.

Andererseits belasten wieder andere, noch kaum beherrschbare schwere Krankheiten, wie Aids, Creutzfeld-Jakob, Alzheimer, zunehmende allergische Krankheiten und die bekannten Zivilisationsschäden aus Bewegungsmangel, Übergewicht, Stress, Strassenverkehr, Süchte und vieles mehr, unser Gesundheitsbudget. Auch ist ein erheblicher Nachholbedarf in der medizinischen Versorgung von Immigranten aus fernen Ländern in Rechnung zu stellen.

Die Umsetzung des medizinischen Fortschritts setzt aber nicht nur fortbildungswillige Ärzte, sondern auch qualifiziertes, motiviertes und entsprechend bezahltes Personal in Spital und Praxis voraus. Ordensschwestern, die früher fast um «Gotteslohn» arbeiteten, sind kaum mehr erhältlich, Gratisleistungen von Familie und Nachbarschaft werden seltener und öffentliche Dienste müssen bezahlt werden. Technische Fortschritte zwingen zu häufigem Ausbau oder zum Ersatz von Apparaten und Einrichtungen.
Ob all der Klagen über die steigenden Behandlungskosten wird meist übersehen oder doch zu wenig berücksichtigt, dass mit den heutigen Leistungen in Spital und Ambulanz dem Patienten nicht nur eine bessere Lebensqualität, sondern den Versicherungen schliesslich auch ein markanter Rückgang in der Dauer von Hospitalisation, ambulanter Behandlung und Arbeitsunfähigkeit oder Invalidität als ökonomische Gegenleistung geboten werden kann.

Die Ansprüche der Gesellschaft und damit der Patienten an die Medizin steigen nicht nur mit den gebotenen Möglichkeiten und der laufend ergänzten und jedermann zugänglichen Information oder «medialen» Indoktrination, sondern eben auch durch den heutigen Lebensanspruch oder das neue Lebensgefühl einer Gesellschaft im modernen Zivilisationswohlstand mit seinem enormen Angebot an Gütern und Möglichkeiten. Bald ist ja «alles» erlaubt und erhältlich. Traditionelle patriarchalisch oder familiär gesetzte Hemmungen sind weitgehend gefallen. Und nicht wenige haben Mühe mit einer freiwilligen Beschränkung im Lebensgenuss und lassen keine Möglichkeiten aus, von extremer Immobilität bis zum Extremsport, von sektiererischen Ernährungsmodellen bis zu Masslosigkeit im Essen und Trinken und bis hin zu allen möglichen Suchtverhalten.

Die weit entwickelte Zivilisation und Verstädterung lässt eben auch die uns angeborenen animalischen Triebe zu Nahrungssuche, zu Sexualität und zu Aggression nicht selten in Perversionen auslaufen, die wiederum nach notwendigen Auffangleistungen rufen.

Heutiger Arbeitsrhythmus, Mobbing, Betriebsamkeit und Unruhe, Umweltstress, soziale Isolation bei fehlendem familiärem oder Gruppenhalt, Schwund religiöser oder autoritärer Bindungen, vermehrte Scheidungen, «broken homes», mangelhafte Erziehung usw. können zu psychosomatischen oder psychischen Störungen führen. Auffallend stark im Steigen ist auch die Zahl depressiver Menschen, was alles zusammen, mit fast pastoralen Anforderungen, einen zeitlich enormen ärztlichen oder fachmännischen Einsatz verlangt.

Aus einer «Feuerwehrmedizin» früherer Jahre, die nur für «ernsthafte» Fälle beansprucht wurde, ist ein Gesundheitscoaching von der Zeugung bis zum bitteren Ende geworden, das für jede Befindlichkeitsstörung ebenso wie für lebensbedrohliche Situationen zur Verfügung stehen muss.

Für unser letztes Lebensviertel insbesondere, soll es noch lebenswert sein, sind Fragen von Gesundheit und Krankheit ganz zentral und wichtig geworden. 
Um den ärztlichen und pflegerischen Bereich haben sich aber auch Hilfstruppen etabliert, die nicht mehr wegzudenken sind, wie Physiotherapeuten, Hauspflegedienste, Mütterberatung, Diätberaterinnen bis hin zu Sozialarbeitern und noch manch andern Wellnesssparten, deren Dienste in Anspruch genommen werden dürfen.

In mancher Hinsicht sind Perfektionismus und Überangebote nicht zu bestreiten. Der Patient ist eben auch kritischer geworden, er akzeptiert nur das Beste; und, wenn selbst betroffen, sogar jene, die alle anderen zu Beschränkung und Verzicht aufrufen. Haftpflichtansprüche an Spital und freie Praxis sind nicht mehr so selten, was entsprechenden Aufwand für medizinische Leistung und Sicherung erzwingt.

Als kostentreibend wird, insbesondere von der Seite der Versicherer, die wachsende Zahl der Ärzte in freier Praxis angeschuldigt, von denen jeder seinen guten Anteil am «Kuchen» haben möchte.

Viele unter uns haben zwar noch die 70er Jahre erlebt, wo die Bevölkerung gewisser Regionen lautstark nach Ärzten gerufen hat und subito ausländische Ärzte (Ostflüchtlinge) ohne eidgenössisches Diplom installiert werden mussten.

Heute scheint der Bedarf an Ärzten offenbar gedeckt, wenn auch noch nicht in sämtlichen Disziplinen. Berücksichtigt man nämlich die vorerwähnten, immer noch wachsenden medizinischen Aufgaben, lehnt man eine 5-Minutenoder Laufbandmedizin ab, und möchten die Ärzte statt 60 bis 70 Stunden pro Woche gar nur noch deren 50 oder weniger arbeiten und den Rest für Entspannung oder für die so notwendige Fortbildung einsetzen, dann kann man auch jetzt bereits nicht mehr von einer Ärzteplethora sprechen. Man darf hier auch daran erinnern, dass bis zu 30\% ausländische Ärzte in schweizerischen Spitälern arbeiten und dort unser eigener akademischer Nachwuchs eher bescheidene Ausmasse hat.

Schwerwiegender ist der Vorwurf, Ärzte in der freien Praxis missbrauchten die soziale Krankenversicherung und verursachten vermeidbare Kosten durch «Mengenausweitung», unzulässige Ausschöpfung des Tarifs oder gar durch Betrügereien.

Es gibt sicherlich Fälle von Überarztung und Tarifausschöpfung bis hin zu betrügerischem Verhalten. Solche Fälle ärgern korrekte Ärzte selber am meisten, weil ja allfällige Vorwürfe, meist in diffuser Art, immer die ganze Ärzteschaft treffen. Konkret werden eben kaum je Namen oder
Zahlen genannt. Der objektive Nachweis eines Tarifmissbrauchs ist auch nicht leicht zu führen. Ärztliche Arbeit hat in der Regel keine Zeugen. So bleibt eben nur der Weg, gegenüber Mittelwerten dauerhaft und wesentlich erhöhte Kosten vorerst einmal zum Anlass zu nehmen, ein Praxisgebaren zu überprüfen. Es müssen dabei im Vergleich zu Mittelwerten richtigerweise auch die medizinische Ausrichtung und Qualifikation, die Leistungen und Einrichtungen des Praxisinhabers, veranlasste Kosten auswärts oder Spitaleinweisungen und auch das «Patientengut» (alt/jung usw.) berücksichtigt werden. Kostenüberschreitungen brauchen keineswegs beabsichtigt zu sein. Schon eine Polypragmasie, das heisst Vielgeschäftigkeit im Ausprobieren verschiedener Behandlungen und Medikamente, kann begründet sein in der Unsicherheit und Ängstlichkeit des Arztes, auch im Perfektionismus oder in der Scheu vor Haftpflichtfolgen.

Im übrigen kann festgestellt werden, dass ambulante Behandlung im Spital oder in der Poliklinik meist kostspieliger ist als in der freien Praxis, obwohl dort für Oberärzte oder Assistenten keine finanziellen Anreize bestehen.

Die Folgerungen aus den erwähnten Kostenfaktoren kann ich vom Kollegen Streit wörtlich übernehmen: «Es wird nie gelingen, die Teuerung im Gesundheitswesen auf das Mass der allgemeinen Teuerung herabzudrücken ohne einschneidende Verzichte, die bis zu einer Rationierung gehen müssten.»

Es bleibt jedenfalls die Erkenntnis, dass die Kosten für das Gesundheitswesen, ein früher für den Kassenversicherten bescheidener Posten im Monatsbudget, zu einem das Leben oder Überleben sichernden Aufwand geworden sind von etwa gleicher Bedeutung wie die existentiellen Aufwendungen für Wohnen, Nahrung, Schule und Ausbildung. Solcher Aufwand scheint mir auch ohne Rationierung immer noch tragbar, wenn man dem die Lebenskosten bestimmenden Warenkorb für das Jahr 2000 entnehmen darf, dass die Kosten für die «Gesundheitspflege» mit 13,4\% jene für «Freizeit und Kultur» $(10,3 \%)$, für «Restaurants und Hotels» $(9,5 \%)$ und für «Verkehr» $(9,4 \%)$ nur mässig übersteigen, wobei diese beiden letzten existentiell doch bedeutend weniger bedrängend scheinen.

Im übrigen sehen wir ähnlich verlaufende Kostensteigerungen in Ländern mit dirigistischen politischen Systemen und ohne Gewährleistung eines ausreichenden Gesundheitsdienstes. 


\section{Mögliche Massnahmen}

Das gleichsam schicksalhafte Ansteigen der Gesundheitskosten soll aber keinesfalls davon abhalten, überall dort Einsparungen zu machen und neue oder andere Lösungen zu suchen, wo dies möglich und zumutbar ist.

$\mathrm{Zu}$ Änderungen im Spitalbereich kann ich mich, mangels eigener Kompetenz, nur in Andeutungen oder Fragestellungen ergehen. Immerhin ist naheliegend, dass im Blick auf Rationalisierung und grösstmögliche Auslastung der Einrichtungen, bei offensichtlich zunehmendem Mangel an Pflegekräften, kommenden Tarifangleichungen und auch angesichts der grösseren Mobilität der «Kundschaft» interkantonal Schwerpunktspitäler - nicht Mammutspitäler - anzustreben sind mit emanzipierten und liberalisierten Führungsstrukturen bis hin zum Einbezug wettbewerbsfördernder privater Anstalten.

In der Praxis machen wir immer wieder die Erfahrung, dass private Labor- und Röntgeninstitute kostengünstiger und kundenfreundlicher arbeiten als kantonale Anstalten.

Zur Effizienzsteigerung der medizinischen Leistungen auf beiden Seiten trüge auch eine grössere Durchlässigkeit zwischen freier Praxis und Spital bei, einerseits durch Zuzug qualifizierter Belegärzte aus der Praxis, andererseits durch Delegierung oder Abkommandierung von Assistenten aus der Klinik in die erfrischende Luft der Praxis.

$\mathrm{Zu}$ gesellschaftspolitischen und allgemein präventiven Massnahmen muss wieder daran erinnert werden, dass man das Fell des Bären nicht waschen kann, ohne es nass zu machen, und es ist voraussehbar, dass ich mit einigen Anregungen nicht nur anstossen, sondern sogar lauten Protest auslösen werde. Aber entweder deklamieren wir weiter: «Wir müssen die Kosten in den Griff bekommen!», oder wir versuchen es dort, wo es möglich und zumutbar ist.

Es wäre dies anzustreben durch: eine ausreichende, zumindest voll schadendeckende Besteuerung der «legalen» Genussgifte und ein Verbot öffentlicher Werbung für dieselben, eine Humanisierung im weitesten Sinne und soweit wie möglich der Arbeitsplätze, der Wohn- und Umgebungsverhältnisse, insbesondere in Städten, Durchsetzung einer besseren Verkehrsdisziplin, Förderung von sinnvoller Freizeitgestaltung und Sport, Prämienbelastung gefährlicher Sportarten, frühzeitige Fürsorgemassnahmen bei drohendem Alkoholismus oder Drogenabhängigkeit und bei Erziehungsunfähigkeit von Eltern.

Die Tatsache, dass immer mehr alte, schwerstkranke, leidende, demente oder komatöse $\mathrm{Pa}$ - tienten dahinvegetieren und einen enormen pflegerischen Aufwand auslösen, für den bei uns nur noch mit Mühe Pflegekräfte gefunden werden können, ist bekannt.

In völlig aussichtslosen terminalen Phasen hat man sich zu passiver Sterbehilfe (Euthanasie), das heisst Unterlassung lebensverlängernder Behandlung, durchgerungen, und auch eine indirekt aktive Sterbehilfe, das heisst Schmerzoder Unruhedämpfung mit möglicherweise tödlichem Ausgang, ist heute akzeptiert.

Einer aktiven Euthanasie durch den Arzt, auf Verlangen eines Patienten, der sich in aussichtsloser, schmerzreicher oder würdeloser Situation befindet (gemäss Motion Cavalli), wurde kürzlich im Parlament keine Straffreiheit zuerkannt.

Immer unter der Voraussetzung, dass alles vorgekehrt wird, um dem Patienten das Weiterleben noch erträglich zu machen, scheint mir diese Ablehnung eine Anmassung gegenüber einer freien menschlichen Entscheidung zu sein.

Gedanken zur aktiven Euthanasie darf man sogar weiter spinnen bis zum holländischen «Modell», wo ein Gremium, gebildet aus Arzt, Pflegekraft und Spitalgeistlichem, straflos über Beendigung eines nur noch sinnlos vegetierenden Lebens beschliessen kann, und dies sogar «ohne» Verlangen des Patienten.

Rein rational und humanitär lässt sich ein solches Vorgehen vertreten, da damit ja auch Mittel und pflegerische Kräfte frei würden für sinnvollere Aufgaben. Widerstand wäre allerdings zu erwarten von religiöser, aber wohl auch von ärztlicher Seite, weil der Arzt, der Lebenserhaltung verpflichtet, auch zum Töter werden müsste.

Eine Rundfrage in der Bevölkerung würde aber mehr Zustimmung für die Beendigung des eigenen sinn- und würdelosen Lebens aufzeigen, als dies wohl manche Ethiker vermuten.

Einsparungen in der freien Praxis sind möglich durch:

Forderung und Förderung ärztlicher Fortbildung. Nur ein gut ausgebildeter Arzt arbeitet effizient und kostengünstig.

Beschränkung der Grundversicherung auf das Notwendige. Notwendig definiert sich als dem Fall angepasste, wissenschaftlich begründete und effiziente Behandlung, die auch wirtschaftlich noch vertretbar sein muss. Zusätzliche Versicherungswünsche könnten mit Prämienzuschlag erfüllt werden.

In diesen zuschlagspflichtigen Bereich müssten eben auch verschiedene Formen alternativoder komplementärmedizinischer Behandlungen verwiesen werden. Wirksame physikalische und pflanzliche Anwendungen sind schon lange in 
die sogenannte Schulmedizin übernommen worden, die immer offen bleiben muss für neue Erkenntnisse und Möglichkeiten.

Als «Pseudo-Naturheilverfahren» müssen aber gemäss Kollegen Roland A. Leemann [3], einem wohlwollend kritischen Kenner und Beurteiler, unter den etwa 200 Naturheilverfahren auch die gegen 40 Varianten der Homöopathie, Formen der Akupunktur und Akupressur, Bioresonanztherapie usw. bezeichnet werden. Nach Aussagen von Krankenkassenseite ist es eben nicht selten, dass Patienten oder deren Ärzte wissenschaftliche und alternative Medizin kumulativ anwenden, was natürlich zu Kostensteigerung führt.

Wenn nun auf diese Beurteilung lauthals protestiert und auf die Wirksamkeit all dieser Behandlungsmethoden hingewiesen wird, darf man bescheiden entgegenhalten, dass bekanntlich 70-80\% aller Krankheiten oder Befindlichkeitsstörungen meist spontan heilen oder erträglich werden, 10-15\% unbedingt ärztlicher Hilfe bedürfen und der Rest bis auf weiteres nicht heilbar ist. Aus langer Erfahrung darf man hier auch zur etwas salopp formulierten Erkenntnis stehen: «Wenn Alternativmedizin 14 Tage lang ausfällt, fällt dies kaum einem Menschen auf, fällt aber die Schulmedizin nur einen Tag aus, wird dies zur landesweiten Katastrophe.»

Die Arzttarife in der sozialen Grundversicherung wurden seit etlichen Jahren nicht mehr dem Lebenskostenzuwachs angepasst. Das mittlere, allerdings aus einer erheblichen Streuung resultierende Reineinkommen des Arztes kann bei etwas über 200000 Franken angesiedelt werden, was im Hinblick auf den späteren Eintritt ins Erwerbsleben und die allein zu erbringende Altersvorsorge in etwa dem Einkommen eines Mittelschullehrers entspricht. Derzeit steht ein Einheitstarif für sämtliche ärztliche Leistungen und für alle Kantone vor der Einführung. Er soll in seinen Auswirkungen, bezogen auf den heutigen Stand, kostenneutral sein, das heisst, er soll trotz allfällig weiterer Zunahme der Zahl der Ärzte oder neuer Behandlungsmöglichkeiten die Gesamtkosten der ambulant erbrachten Leistungen nicht erhöhen. Schwierigkeiten sind hier unschwer vorauszusehen.

Für die fakultativen Zusatz- oder Privatversicherungen, die tatsächlich ausgebeutet werden können, wäre zu fordern, dass hier die zuständigen Fachgesellschaften ihre Tarife mit vertretbaren und verbindlichen oberen Rahmenansätzen belegen, deren Beachtung von Patienten und Versicherungen eingeklagt werden könnte.

Es ist dem Bild des Arztes, der schliesslich von Krankheit und Leid des Menschen lebt, unzuträglich, wenn er sein Einkommen demjenigen gewisser Spitzensportler oder Wirtschaftsmanager anzunähern sucht.

Für mich ist eigentlich unverständlich, dass das Angebot paritätisch gewählter Vertrauensärzte oder Kommissionen von den Versicherungen so wenig genutzt wird und meist nur ungezielte generelle Vorwürfe erfolgen. Disziplinierend für beide, Versicherer wie Übeltäter, wäre die Forderung, jeweils auch Namen und Zahlen zu nennen. Erhöhte Arztkosten sind allerdings, wie erwähnt, noch kein Beleg für Missbrauch, dürfen aber Anlass für Abklärungen sein.

Offenbar zur Disziplinierung der ansässigen und mit Blick auf den Zustrom der ausländischen Ärzte aus dem EU-Raum schlägt das Parlament (vorerst der Ständerat) die Aufhebung des Kontrahierungszwanges für Krankenversicherungen vor. Ein verständlicher Gedanke in den Anfängen der sozialen Krankenversicherung mit noch 20-30\% Versicherten, nicht aber unter dem Regime der obligatorischen allgemeinen Volksversicherung und insbesondere dann, wenn die Versicherer allein darüber befinden könnten, mit wem sie sich vertraglich binden oder lösen wollen.

Da ja anzunehmen wäre, dass nicht nur einzelne Kassen, sondern eben alle im Verbund der santésuisse, auch ohne offizielle Absprache, gegenüber missliebigen Ärzten logischerweise zum gleichen Schritt bewegt würden, käme dies für die Betroffenen einem faktischen Berufsverbot oder glatten Entzug der Existenzgrundlage gleich, da ja noch immer nicht ausreichend arabische Scheichs kompensierend einspringen könnten. Abgesehen von einer empfindlichen Einschränkung der freien Arztwahl wäre auch die finanzielle Ausbeute recht bescheiden angesichts der Tatsache, dass die Arztkosten in der freien Praxis etwa 14-15\% der Gesamtkosten beschlagen und die jenen Ärzten entzogene Arbeit schliesslich von den übrigen, wenn auch kostengünstiger, geleistet werden müsste.

Im Status des Versicherungsobligatoriums und in der geplanten willkürlichen Anwendung ist dieses Vorhaben inakzeptabel. Zwar nicht tröstlich, aber doch erheiternd zu vernehmen wären ja wohl auch die lautstarken Reaktionen von Vertretern anderer Berufsgruppen oder gar von Parlamentariern, wenn man ihnen solcherart ans «Eingemachte» ginge.

Auch die Ärzteschaft hat Interesse an einer Disziplinierung ihrer «schwarzen Schafe», aber nur unter der Voraussetzung, dass unter den gegebenen Bedingungen eine Massnahme von dieser Tragweite nur in Einzelfällen und durch eine paritätisch zusammengesetzte Kommission aus Vertretern der Versicherungen, der Ärzte und 
der Patienten, und nach vorausgegangener «Frostwarnung», beschlossen würde.

Die Aufwendungen für Medikamente erreichen bei uns eine Höhe von gegen 4 Milliarden. Die Pharmaforschung ist kostspielig und rechnet in der Regel mit einem geldsegenbringenden Volltreffer auf etwa 100 Versuche oder Präparate. Und immer wieder verdrängt das noch Bessere das Gute. Das ist richtig so, weil das Optimum immer auch am preisgünstigsten ist.

Die Pharmaindustrie floriert jedenfalls und fährt derart hohe Gewinne ein, dass es durchaus vertretbar ist, die Höhe der Medikamentenpreise nicht einfach ergeben hinzunehmen, sondern zu hinterfragen und Vergleiche anzustellen.

Die «Denner-Initiative für kostengünstigere Präparate» des letzten Jahres hat, leider in missverständlicher und laienhafter Form, versucht, Parallelimporte der in den umliegenden Ländern deutlich billigeren Produkte der gleichen Produzenten und den vermehrten Einsatz von identischen Nachahmerpräparaten (Generika) durchzusetzen. Unter dem Trommelfeuer der Pharmaindustrie und ihr zugetaner Politiker und Ärzte ist sie gescheitert. Inzwischen sind die damals «zweifelhaften» Generika aber schon weitgehend salonfähig geworden, und die Margen der Verteiler konnten, zumindest bei teuren Medikamenten, dank gewährter Kompensationen gesenkt werden. Ob dies zum Schluss Einsparungen ermöglicht, bleibt abzuwarten.

Einsparungen erbrächten hier sicherlich eine stärkere Sensibilisierung der Patienten (und Ärzte) für die Kosten der Medikamente. Der Patient, der sich nicht spontan dafür interessiert, hat in der Regel keinerlei Einsicht in diese Preise. Die Abrechnung läuft ja direkt über die Krankenkasse. Nur so ist eigentlich verständlich, dass auch mit teuren Medikamenten oft recht sorglos umgegangen wird, sie auch nicht oder nicht richtig eingenommen, weggeworfen, unnötigerweise in Grosspackungen oder mehrfach bezogen wer- den, usw. Gelegentliche Kontrolle des Medikamentenkästchens fördert da oft Erstaunliches zutage. Willfährigkeit (heute: compliance) und Sparsamkeit in der Anwendung von Medikamenten könnten eindeutig gefördert werden dadurch, dass der Patient jedesmal oder periodisch seine Medikamente aus der eigenen Tasche bezahlen müsste und Rückerstattung von seiner Versicherung erhielte; also mit dem System des Tiers garant, wie dies für die meisten Arztrechnungen gehandhabt wird, um dem Patienten Einsicht in die Kosten zu geben und seine Mündigkeit zu respektieren. Mit einer solchen Sensibilisierung des «Konsumenten» könnten schätzungsweise Einsparungen von 10\%, also gegen 400 Millionen Franken, erzielt werden.

Widerstand gegen eine solche Änderung wäre wohl von seiten der Versicherer wegen vermehrter Umtriebe und von seiten der Apotheker wegen Furcht vor Umsatzeinbussen zu erwarten.

Aus nur wenig beeinflussbaren Gründen werden die Kosten im Gesundheitswesen wohl weiterhin steigen. Und nur über Offenlegung der Ursachen und der vermeidbaren Faktoren, über Kostentransparenz und Appell an Mündigkeit und Mitverantwortung aller Beteiligten und daraus abzuleitenden tragbaren Verzichten oder Einschränkungen sind auch in diesem schwerlastigen Gesundheitswesen noch Einsparungen zu machen.

\section{Literatur}

1 Streit R. Warum steigen die Kosten im Gesundheitswesen überproportional an? Schweiz Ärztezeitung 2000;81(40):2263-7.

2 Oberle A. Von den Kosten im Gesundheitswesen. In: Aspekte des Aargauischen Gesundheitswesens. Bd 1. Aarau; 1978. S. 49-54.

3 Leemann RA. Schulmedizin/Alternativmedizin. Wetzikon: Buchverlag; 1997. 